\title{
A monolithic hydro/organo macro copolymer actuator synthesized via interfacial copolymerization
}

\author{
Feilong Zhang ${ }^{1,3}$, Junbing Fan ${ }^{2}$, Pengchao Zhang ${ }^{2}$, Mingjie Liu ${ }^{4}$, Jingxin Meng ${ }^{2}$, Lei Jiang ${ }^{2,3,4}$ \\ and Shutao Wang 2,3
}

Synthetic polymer actuators have attracted increasing attention for their potential applications in artificial muscles, soft robotics and sensors. The majority of previous efforts have focused on smart hydrogels with bilayer structures that can change their shape in response to environmental stimuli, such as temperature, light and certain chemicals. However, the practical application of hydrogels is limited because of their low modulus and weak mechanical strength. Here we synthesized a robust monolithic actuator of a macro-scale hydro/organo binary cooperative Janus copolymer film. The process involves direct, one-step interfacial polymerization of immiscible hydrophilic and hydrophobic vinyl monomer solutions, and the resultant product exhibited binary cooperative shape transformation to multiple external stimuli. The Janus copolymer film can work in both aqueous solutions and organic solvents, with bidirectional and site-specific bending arising from cooperative asymmetric swelling/shrinking of the hydrogel and organogel networks. In addition, the as-prepared Janus copolymer film can act as a sensor element for solvent leakage detection. This binary cooperative strategy is applicable to most immiscible monomer systems and provides a general approach to developing novel functional copolymer materials.

NPG Asia Materials (2017) 9, e380; doi:10.1038/am.2017.61; published online 19 May 2017

\section{INTRODUCTION}

The shape transformations of biological organisms ${ }^{1-6}$ have been the inspiration for many products in the field of artificial muscles, ${ }^{7-13} \mathrm{soft}$ robotics, ${ }^{14-19}$ sensors $^{20}$ and complex shape engineering. ${ }^{21,22}$ For example, the leaves of the Venus flytrap snap together to capture insects by virtue of the synergy between the hydroelastic instability and asymmetric expansion of the inner and outer surfaces at the cellular level. $^{2}$ The layered anisotropic orientated cellulose fibrils induce hygroscopic movements of pine cones, ${ }^{1}$ wheat awns, ${ }^{3}$ orchid tree seedpods ${ }^{6}$ and other plants. By mimicking the sophisticated hierarchical structures present in nature, the motion of polymer films has been successfully demonstrated in several cases. ${ }^{23-30}$ For example, hydrogel bilayers embedded with intersecting inorganic platelets or cellulose fibrils have exhibited pine-cone-like bending and pod-like twisting motions. ${ }^{24,31}$ However, the practical applications of these actuators were limited because of the low modulus and weak mechanical strength of the hydrogels. ${ }^{32}$ Elastomer single layer films, such as azobenzene polymer films synthesized using an elaborate molecular design, ${ }^{13,27}$ can achieve smart responsive curving. However, these films require a unidirectional stimulus to generate anisotropic contraction/expansion of their two sides; this factor limits the film thickness to the micrometer or sub-millimeter level. ${ }^{27,33-35}$ Therefore, there is great opportunity and challenge in exploring new strategies to synthesize robust intelligent polymer actuators.

Amphiphilic block copolymers exhibit unique and fascinating locomotion by virtue of the different hydrophilic/hydrophobic properties of the two segments in the polymer chains. ${ }^{36}$ For example, the binary cooperative effect ${ }^{37,38}$ of the hydrophilic segments and hydrophobic segments cause the polymer chains to assemble into aggregates in aqueous solutions, with the hydrophobic segments twisting and coiling to entangle each other via hydrophobic interactions as the core. ${ }^{39}$ Under certain conditions, the aggregates are able to transform among various morphologies, ${ }^{39-42}$ including spheres, rods, ${ }^{43}$ vesicles, ${ }^{44}$ tubules ${ }^{45}$ and cubosomes ${ }^{46}$ similar to actuators. Therefore, it seems feasible to combine a hydrogel with an organogel to fabricate a covalently connected macro-scale 'amphiphilic diblock copolymer aggregate,' whereby the two subunits of diverse physical and chemical properties realize macroscopical motion. Here we report the fabrication of a macro-scale binary cooperative hydro/organo copolymer film by interfacial polymerization with an aqueous monomer solution and an organic monomer solution. This hydro/organo copolymer film exhibits intelligent multi-responsive shape transformations (Figure 1). The Janus copolymer film is composed of a hydrogel network

${ }^{1}$ Beijing National Laboratory for Molecular Sciences (BNLMS), Key Laboratory of Green Printing, Institute of Chemistry, Chinese Academy of Sciences, Beijing, China; ${ }^{2}$ Key Laboratory of Bio-inspired Materials and Interfacial Science, CAS Center for Excellence in Nanoscience, Technical Institute of Physics and Chemistry, Chinese Academy of Sciences, Beijing, China; ${ }^{3}$ University of Chinese Academy of Sciences, Beijing, China and ${ }^{4}$ Key Laboratory of Bio-Inspired Smart Interfacial, Science and Technology of Ministry of Education, School of Chemistry and Environment, Beihang University, Beijing, China

Correspondence: Professor S Wang, Key Laboratory of Bio-Inspired Materials and Interfacial Science, Technical Institute of Physics and Chemistry, Chinese Academy of Sciences, 29 Zhongguancun East Road, Haidian District, Beijing 100190, China.

E-mail: stwang@mail.ipc.ac.cn

Received 26 September 2016; revised 14 February 2017; accepted 28 February 2017 


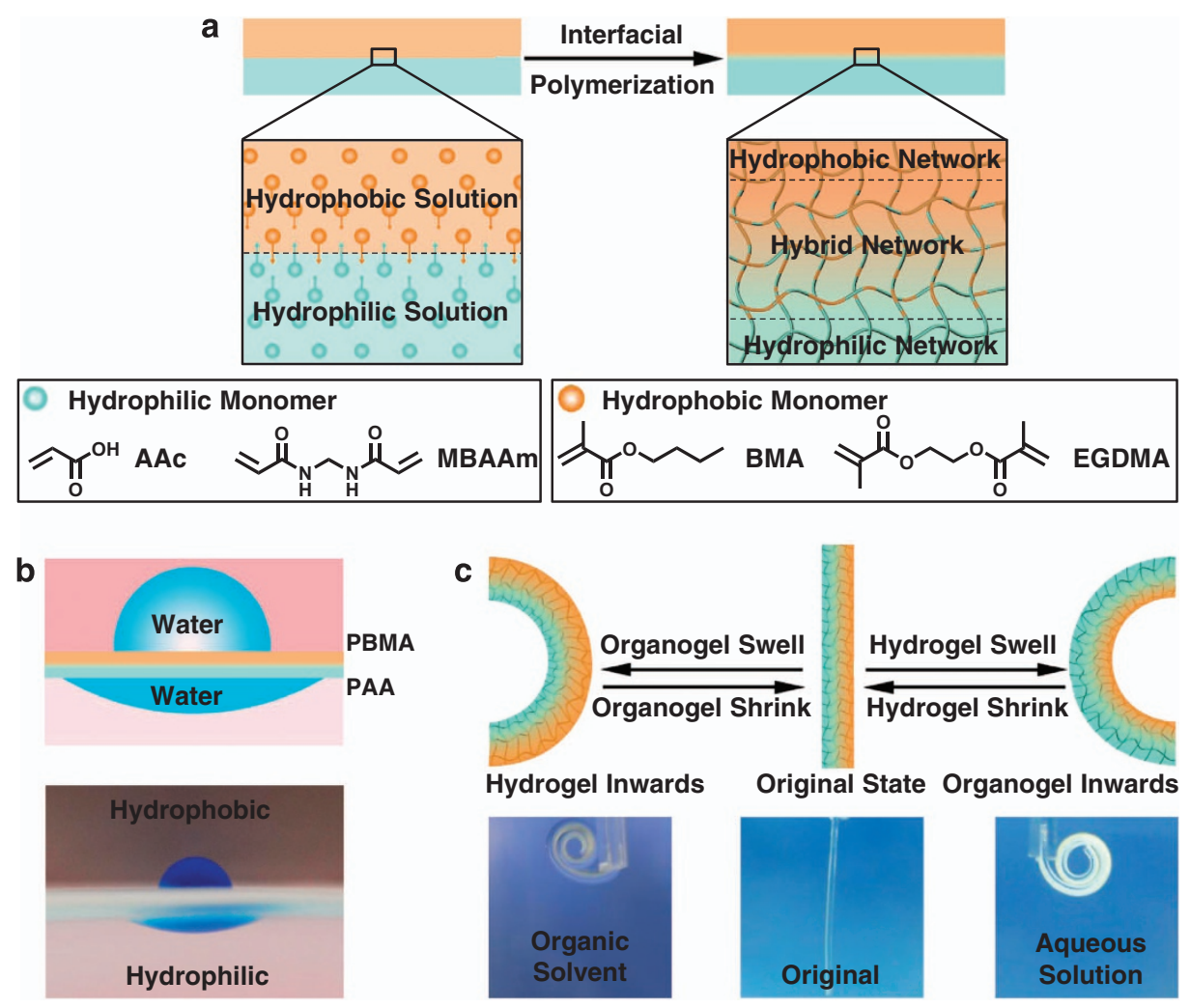

Figure 1 Design of shape-transformable hydro/organo Janus copolymer films. (a) A schematic showing the Janus film synthesized via interfacial polymerization of hydrophobic and hydrophilic vinyl monomer solutions. (b) Different wettability of water on the two surfaces of the Janus film. The upper surface (PBMA network) exhibits higher hydrophobicity than the lower surface (PAA network); schematic (top) and optical image (bottom). It is noteworthy that water is stained blue with methylene blue. (c) Bidirectional bending under the binary cooperative effect of the hydrogel network and the organogel network. Immersed in aqueous solution (proper pH value), the hydrogel network of the Janus film is solvated and undergoes expansion, leading to bending of the Janus film with the organogel network inwards. The Janus film bends with hydrogel network inwards when immersed in organic solvents (acetone), due to the swelling of organogel network.

(low modulus) and organogel network (high modulus), similar to the hydrophilic and hydrophobic chains in amphiphilic diblock copolymer aggregates. Under the binary cooperative effect of the hydrogel and organogel networks, unidirectional, bidirectional and site-specific bending were realized by the Janus copolymer film in aqueous solutions, organic solvents and mixed solvent systems. All these shape transformations originate from the binary cooperative effect of the hydrogel and organogel networks in the copolymer film with different expansion coefficients. In addition, the binary cooperation between the hydrogel and organogel networks allows the Janus copolymer film to function as a smart sensor element for solvent leakage detection.

\section{EXPERIMENTAL PROCEDURE}

\section{Materials}

Acrylic acid 99\% (AA, Lot number LC80O82), acrylamide 99\% (Lot number L510P42), $N, \quad N^{\prime}$-dimethylacrylamide $98 \%$ (Lot number LT60N21), $N, N^{\prime}$-methylenebis(acrylamide) $98 \%$ (Lot numberLT50N18), ammonium persulfate $98 \%$ (Lot number LBBON03), $n$-butyl methacrylate 99\% (BMA, Lot number LT60P86), styrene 99.5\% (Lot number LT40O46), methyl methacrylate 99\%, ethylene glycol dimethyl acrylate 99\% (Lot number LA10L18), divinyl benzene $80 \%$ in ethylvinylbenzene and diethylbenzene (Lot number LT40O101) and 2, 2'-azobis(2-methyl-propionitrile) 99\% (Lot number LD30O37) were purchased from J\&K, Beijing, China, and poly(vinyl alcohol) 1788 (Lot number L1409024) was purchased from Aladdin, Shanghai, China. All the reagents were used as received, except the styrene, which was purified using an $\mathrm{Al}_{2} \mathrm{O}_{3}$ column to remove the stabilizer.

\section{Procedures}

Preparation and characterization of the PAA-B-PBMA copolymer films. The copolymer films were fabricated as follows. The aqueous reaction solution was prepared by mixing a $5 \mathrm{~mol}^{-1}$ monomer solution (AA) and a $4 \mathrm{wt} \%$ poly (vinyl alcohol) (thickening agent) aqueous solution (volume ratio $=1: 1$ ) using $N, N^{\prime}$-methylenebis(acrylamide) as the crosslinker (mole ratio of divinyl to vinyl monomers $=1: 200)$ and the ammonium persulfate as an initiator $(10 \mathrm{mg}$ in $1 \mathrm{ml}$ reaction solution). The organic reaction solution consisted of BMA as the monomer, ethylene glycol dimethyl acrylate $\left(6.5 \mu \mathrm{ml}^{-1}\right)$ as the crosslinker and 2, $2^{\prime}$-azobis(2-methyl-propionitrile) $\left(20 \mathrm{mg} \mathrm{ml}^{-1}\right)$ as the initiator. Both reaction solutions were bubbled with nitrogen to remove the dissolved oxygen. Then, a $2.5 \mathrm{ml}$ aqueous reaction solution and a $2.5 \mathrm{ml}$ organic reaction solution were successively pipetted into a glass Petri dish $(6 \mathrm{~cm}$ diameter and treated with $\mathrm{O}_{2}$ plasma before use). After a short standing time, polymerization was carried out at $65^{\circ} \mathrm{C}$, heated in a water bath for $90 \mathrm{~min}$ and the poly AA-Block-poly n-butyl methacrylate (PAA-B-PBMA) network was obtained. For the analysis of the physical and chemical properties, the pure PAA films and PBMA films were fabricated as follows. By injecting the reaction solution into the spacer of two glass slides separated by $0.5 \mathrm{~mm}$-thick copper wire, the individual hydrophilic gel films and hydrophobic films were polymerized under the same conditions as the Janus film fabrication.

To singularize the Janus properties of the prepared films, the PAA-B-PBMA films were treated with an $\mathrm{NaOH}$ solution to transform the $-\mathrm{COOH}$ to -COONa before elemental examination. The distribution of sodium reflects the composition and distribution of PAA in the films. The morphologies of the Janus films were examined using a field-emission scanning electron microscope (JSM-7500F, JEOL, Tokyo, Japan) at $5 \mathrm{kV}$; the samples were coated with a thin layer of gold before examination. The elemental compositions along the cross- 
a
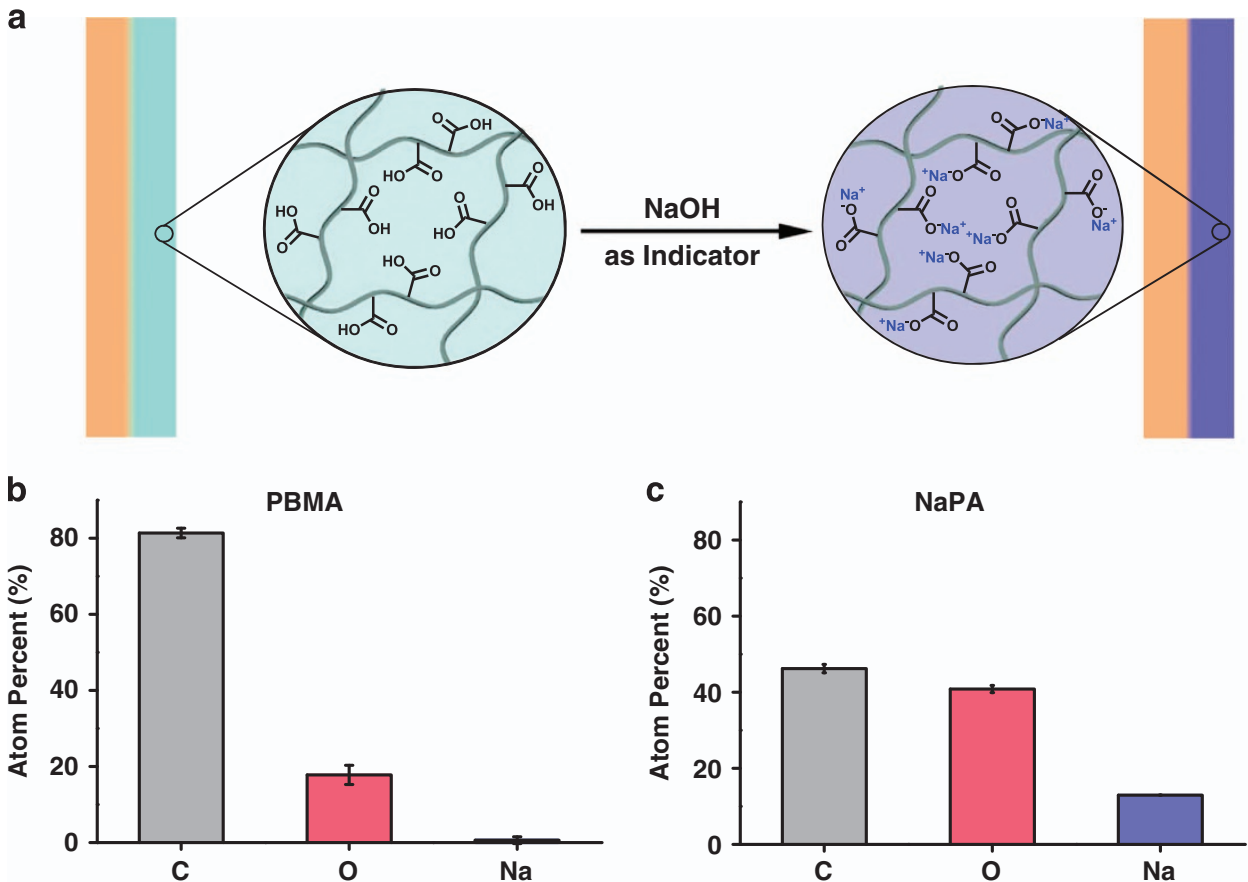

C
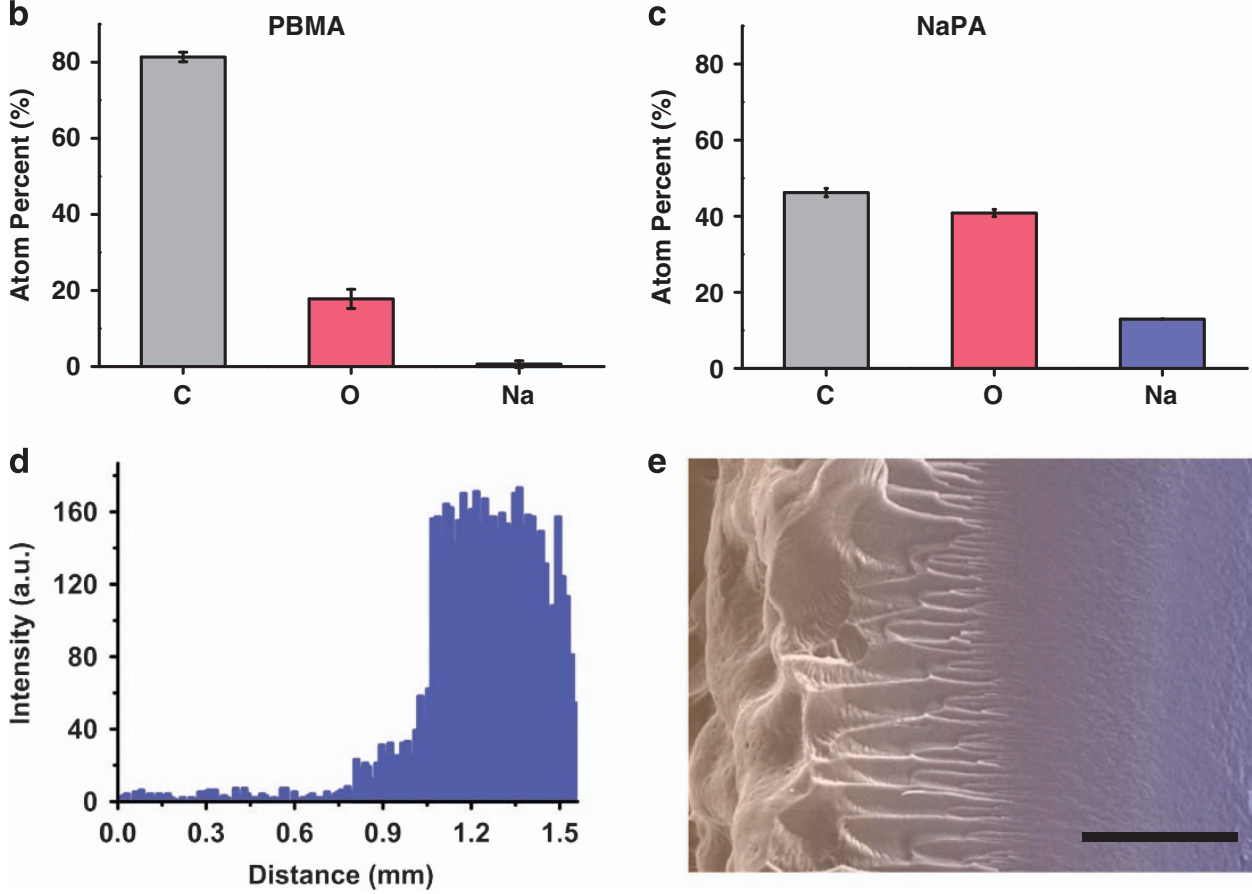

e

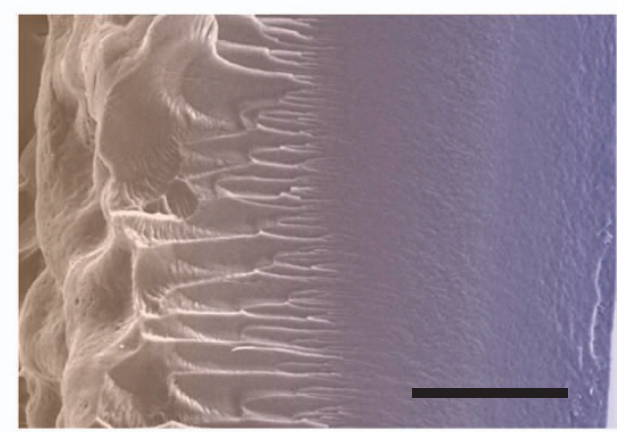

Figure 2 Janus composition and flawless connection of the PAA-B-PBMA copolymer films. (a) To verify the anisotropic property of the film, the film was treated with $\mathrm{NaOH}$ to deprotonate the $\mathrm{COOH}$ into $\mathrm{COO}^{-} \mathrm{Na}^{+}$groups. (b and $\mathbf{c}$ ) Elemental composition of the upper (PBMA) (b) and lower (sodium polyacrylate, $\mathrm{NaPA}$ ) (c) surfaces of the $\mathrm{NaOH}$-treated film. (d and e) Cross-sectional view of the Janus film and the intensity gradient of the sodium from the PBMA network to the NaPA network along the film verified that the film was a monolith and flawless. Scale bar, $100 \mu \mathrm{m}$.

section and on both sides of the film were analyzed using energy dispersive $\mathrm{X}$-ray (Oxford Instruments, Oxfordshire, UK) via scanning electron microscope at $15 \mathrm{kV}$.

Variation in the swelling ratio of the linear dimensions of the pure PAA films and PBMA films in an aqueous solution with varying $\mathrm{pH}$ and $\mathrm{C}_{\mathrm{NaCl}}$, organic solvents and acetone-water mixtures. The individual hydrogel films and hydrophobic polymer films were cut into squares with a certain side length, denoted as $d_{0}$. After immersion in a given solution for more than $30 \mathrm{~min}$, the side lengths of the squares were measured and denoted as $d$. The swelling ratio of the linear dimensions of the film was defined as $f=d / d_{0}$. The $\mathrm{pH}$ of the solution was adjusted with $\mathrm{HCl}$ solution or $\mathrm{NaOH}$ solution and measured with a pH meter (FE20K, Mettler Toledo, Zurich, Switzerland).

Shape transformation tests of the Janus copolymer film. Rectangular pieces $(24 \mathrm{~mm} \times 2-3 \mathrm{~mm})$ were cut from the prepared Janus film as actuators. The actuators bend to different degrees in different solutions or solvents. The bending motion of the actuator in a given solution or solvent was recorded using a digital camera. The radius of curvature of the actuators was measured using image-processing software.

\section{RESULTS AND DISCUSSION}

Preparation and shape transformation mechanism of the binary cooperative hydro/organo copolymer films

The hydro/organo Janus copolymer films were prepared from a bilayer solution resulting from the immiscibility of a hydrophilic monomer solution (AA) and a hydrophobic monomer solution (BMA) (Figure 1a). Owing to its greater density, the AA aqueous solution with ammonium persulfate as the initiator was added to a glass Petri dish to spread over the surface. Then, a BMA solution with 2,2'-azobis (2-methyl-propionitrile) as the initiator was added to form the top layer (Figure 1a). After a short standing time, polymerization was initiated by heating in a water bath at $65^{\circ} \mathrm{C}$ and interfacial polymerization of the hydrophobic monomers and hydrophilic monomers occurred at the oil/water interface (Supplementary Scheme S1). After $1.5 \mathrm{~h}$ of polymerization, a free-standing and monolithic Janus copolymer network (with a thickness of $\sim 1.1 \mathrm{~mm}$ ) was obtained and denoted as PAA-B-PBMA, which exhibits similar mechanical properties (tensile strength) to that of the PBMA film and is much tougher than the PAA film (Supplementary Figure S1). The combination of the hydrogel network and organogel 
a
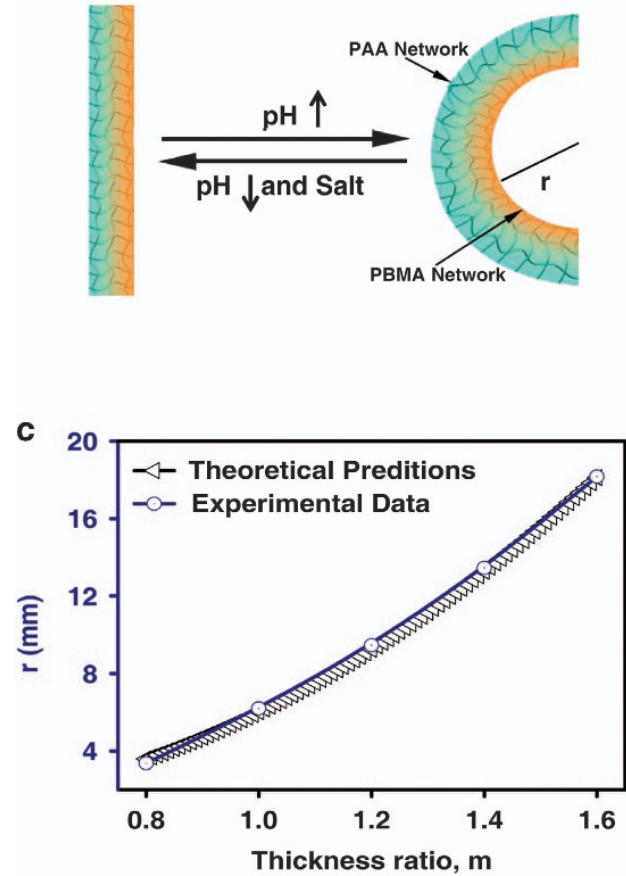

e

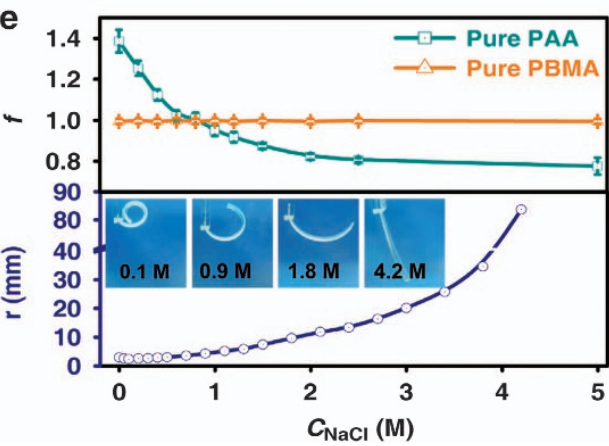

b

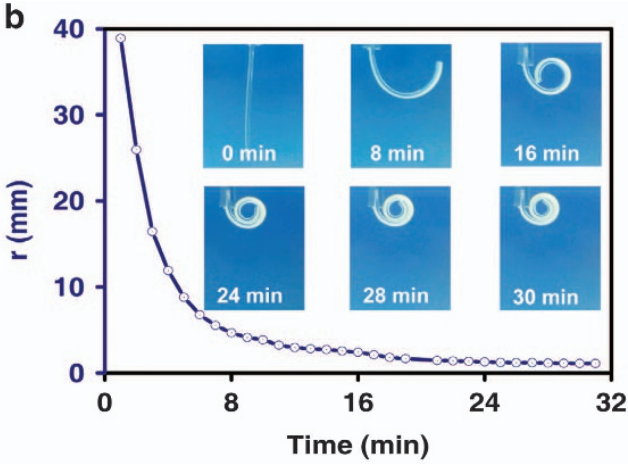

d

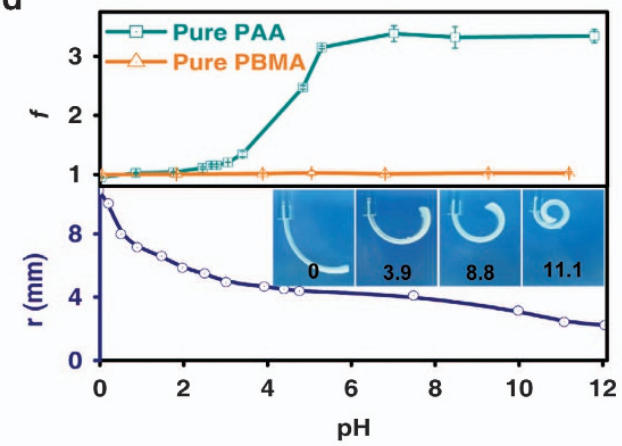

f
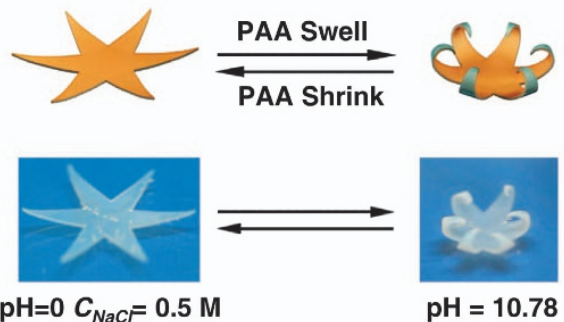

Figure 3 Intelligent unidirectional shape transformation of the PAA-B-PBMA Janus film in an aqueous solution. (a) A schematic of the film coiling and decoiling in aqueous solutions, depending on the swelling and shrinking of the hydrogel network and definition of $r$, the radius of curvature of the coiled film. (b) A typical coiling process of the PAA-B-PBMA Janus film in $\mathrm{pH} 12.8 \mathrm{NaOH}$ aqueous solutions. The film coiled gradually with a decreasing radius of curvature against time, which reached a minimum after $30 \mathrm{~min}$. The inset shows snapshots of the film coiling. (c) Theoretical and experimental plots of the radius of curvature against the volume ratio of the BMA monomer solution to the AA monomer solution in water (pH 6.9). (d) The Janus film curls to a certain extent controlled by the $\mathrm{pH}$ value of the aqueous solution. The top shows the relative change in the linear dimensions, $f$, of the individual pure PAA and PBMA films, plotted as a function of $\mathrm{pH}$. The bottom shows the variation in the radius of curvature of the PAA-B-PBMA Janus film, plotted versus $\mathrm{pH}$. (e) The curled PAA-B-PBMA Janus film stretches gradually with the increase of $C_{\mathrm{NaCl}}$ in the $\mathrm{NaCl}$ aqueous solution. The top shows $f$ for individual pure PAA and PBMA films, plotted as a function of $C_{\mathrm{NaCl}}$. The bottom shows the variation in the radius of curvature of the PAA-B-PBMA film, plotted versus $C_{\mathrm{NaCl}}$. (f) A PAA-B-PBMA 'flower' closes in an $\mathrm{NaOH}$ aqueous solution $(\mathrm{pH} 10.78)$ and re-opens in $1 \mathrm{~m} \mathrm{NaCl}$ aqueous solution (pH 0 ).

network provides the opportunity for the binary cooperation of their complementary properties.

The as-prepared hydro/organo Janus copolymer film exhibited different properties at their two sides, such as wettability, modulus, expansion coefficient and so on. As shown in Figure 1b, the upper surface (PBMA network) exhibited more hydrophobicity than the lower surface (PAA network), which shows similar water contact angles to that of the pure PBMA network and the pure PAA network, respectively (Supplementary Figure S2). The hydrogel and organogel networks are similar to the hydrophilic chains and hydrophobic chains in amphiphilic diblock copolymer aggregates, respectively, because they are both able to act as the active parts of the Janus film. As such, immersed in water, the
PAA hydrogel network was capable of absorbing large amounts of water, resulting in significant volume expansion, whereas the organogel network remained in its original state. The non-uniform internal stresses between the hydrogel and organogel networks coiled the copolymer film, with the organogel network bending inwards (Figure 1c right). When immersed in organic solvents, the PAA-B-PBMA copolymer network bent with the hydrogel network inwards due to the swelling of the PBMA network (Figure 1c left). In addition, the bent Janus film could recover its original shape (straight) when the internal stress was eliminated by varying the conditions. These shape transformations are attributed to the binary cooperative complementary effect of the hydrogel and the organogel networks. 


\section{Janus composition}

The elemental composition of our Janus copolymer film was determined via energy-dispersive X-ray spectroscopy. To help distinguish the Janus properties, sodium was introduced as an indicator by simply soaking the films in an $\mathrm{NaOH}$ aqueous solution for several minutes, during which the $-\mathrm{COOH}$ (in PAA) was deprotonated into $-\mathrm{COO}^{-} \mathrm{Na}^{+}$(in sodium polyacrylate) (Figure 2a). As the $-\mathrm{COOH}$ exists only in PAA, the content and distribution of sodium are consistent with that of PAA. The results showed that sodium was hardly detected in the upper surface (PBMA network) and the atom ratio of $\mathrm{C}$ to $\mathrm{O}$ was $\sim 4.6$ (Figure $2 \mathrm{~b}$ ), which is similar to the calculated value of PBMA (4.0), whereas the atom ratio of $\mathrm{C}, \mathrm{O}, \mathrm{Na}$ was $~ 1.13: 1$ : 0.32 (Figure $2 \mathrm{c}$, the calculated value of sodium polyacrylate was $1.5: 1: 0.5)$ in the lower surface. The sodium content and atom ratio of $\mathrm{C}$ and $\mathrm{O}$ confirmed the Janus composition in the PAA-B-PBMA film.

We visually verified the seamless connection of the hydrogel and organogel networks in the Janus film by scanning electron microscopic imaging and elemental distribution analysis of the cross-section of the $\mathrm{NaOH}$ treated films. No obvious cracks were observed from the scanning electron microscopic images (Figure 2e) and the existing structural gradient confirmed the Janus properties of the film. In the sodium intensity bar chart (Figure $2 \mathrm{~d}$ ), a lower intensity was found at the PBMA network side, whereas a higher intensity existed at the sodium polyacrylate network side and a gradually increasing intensity from PBMA network to sodium polyacrylate network in the middle of the film also verified the Janus property and flawless connection, which was further confirmed by the intensity line plots of $\mathrm{C}$ and $\mathrm{O}$ in Supplementary Figure S3.

\section{Intelligent shape transformation in aqueous solutions}

The binary cooperative effect of the hydrogel and organogel networks endowed the film with the ability to transform in response to environment changes. To explore the shape transformation mechanism and quantify the bending behavior of the Janus film, two parameters were introduced: the swelling ratio in the linear dimension, $f$, and the radiius of curvature, $r$ (Figure 3a). When immersed in an aqueous solution ( $\mathrm{pH}$ 12.6), the flat film $(24 \mathrm{~mm} \times 3 \mathrm{~mm})$ bent gradually into multiple coils with the PBMA surface facing inwards, as the hydration of the PAA network gave rise to non-uniform internal stresses (Figure 3b, inset, Supplementary Figure S4 and Supplementary Movie S1). The radius of curvature $r$ reached a constant value $(\sim 1.1 \mathrm{~mm}$, inner radius $)$ after being immersed for $30 \mathrm{~min}$ in the aqueous solution; thus, the swelling equilibrium was reached (Figure $3 \mathrm{~b}$ ). The bending of the Janus films can be explained using Timoshenko's theory for bimetal thermostats. ${ }^{47}$ The related equation was modified (shown in Supplementary Information section 4) as follows:

$$
r \sim \frac{a_{2}\left(3(1+m)^{2}+(1+m n)\left(m^{2}+\frac{1}{m n}\right)\right)}{6 \Delta f(1+m)}
$$

where $a_{2}$ is the thickness of the PAA network; $m$ is the ratio between the thickness of the PBMA network $\left(a_{1}\right)$ and that of the PAA network $\left(a_{2}\right), a_{1} / a_{2} ; n$ is the ratio of the elastic modulus $(E)$ between the PBMA network and the PAA network $\left(E_{\mathrm{PBMA}} / E_{\mathrm{PAA}}\right)$; and $\Delta f$ is the difference of $f$ between the PAA network and the PBMA network, $\Delta f=f_{\mathrm{PAA}}-f_{\mathrm{PBMA}}$. When immersed in the aqueous solution, the PAA network swelled with time and the PBMA network remained the same, resulting in an increasing $\Delta f$, which decreased the $\mathrm{r}$ as demonstrated in equation (1).
According to equation (1), the radius of curvature $r$ of the bent Janus films can be regulated with the changes of $a_{2}, m, n$ and $\Delta f$ (Supplementary Figure S5). To verify this speculation, the Janus films with different $m$ values were fabricated by varying the ratio of prepolymerization solution volumes between the BMA solution and AA solutions $\left(V_{\mathrm{BMA}}\right.$ and $\left.V_{\mathrm{AA}}\right)$, with $V_{\mathrm{AA}}$ as a constant, resulting in a constant $a_{2}$, and $m \propto V_{\mathrm{BMA}} / V_{\mathrm{AA}}$. When immersed in an aqueous solution (for example, $\mathrm{pH}$ 6.9), the radius of curvature of the bent Janus films was found to increase with increasing $V_{\mathrm{BMA}} / V_{\mathrm{AA}}$ (Figure $3 \mathrm{c}$ and Supplementary Figure S6). This result is similar to that predicted from equation (1) (Figure 3c), within the given scope of $V_{\mathrm{BMA}} / V_{\mathrm{AA}}$ values from 0.8 to 1.6 with a compensation coefficient of 10.5 ( $n \approx 165$, calculated from Supplementary Figure S7, $\Delta f=2.3, a_{2}=0.9 \mathrm{~mm}$ ).

Either network of the Janus film with the ability of stimuli responsiveness could change the above parameters when stimulated, giving rise to variations of the radius of curvature, $r$. The PAA is sensitive to the $\mathrm{pH}$ value of the solution, as the swelling ratio in the linear dimension $(f)$ of the pure PAA films increased with the solution $\mathrm{pH}$ value (0 to 7$)$ and reached equilibrium with a maximum of $\sim 3.3$ (7 to 12) (Figure 3d, top), which was attributed to the deprotonation of the $-\mathrm{COOH}$ groups, leading to repellency of the PAA chains, whereas the $f$ of the pure PBMA films remained unchanged. The resulting increase in the difference of $f$ between the PAA network and the PBMA network $(\Delta f)$ led to the reduction of the radius of curvature of the Janus films $(r)$ (Figure 3d, bottom and Supplementary Figure S8), which can be explained with the help of equation (1) (the variation in the modulus $(n)$ is a second-order effect compared with $\Delta f$ ). Therefore, the bending of the PAA-B-PBMA Janus film can be controlled simply by changing the $\mathrm{pH}$ value of the environment under the binary cooperative effect of the hydrogel and organogel networks.

When placed in an $\mathrm{NaCl}$ solution, the bent Janus films stretched gradually and straightened as the concentration of $\mathrm{NaCl}$ increased, $C_{\mathrm{NaCl}}$, owing to the ionic strength-mediated dehydration ${ }^{48,49}$ of the PAA network. The swollen pure PAA hydrogel films shrunk in the $\mathrm{NaCl}$ solutions and the swelling ratio in the linear dimension $f$, exhibited a sharp decrease for $0 \mathrm{M} \leqslant C_{\mathrm{NaCl}} \leqslant 2.0 \mathrm{~m}$ to a constant value of $\sim 0.8$, whereas that of the PBMA network remained unchanged (Figure $3 \mathrm{e}$, top). Correspondingly, the difference in $f$ between the PAA network and the PBMA network $(\Delta f)$ decreased, resulting in the increase of the radius of curvature of the Janus films, $r$ (Figure $3 \mathrm{e}$, bottom and Supplementary Figure S9). As a supporting validation of the controllability of the Janus film bending, a star-shaped PAA-BPBMA network film exhibited excellent close and open properties, similar to a blooming flower, in a $\mathrm{NaOH}$ solution $(\mathrm{pH} 10.8)$ and a $\mathrm{NaCl}$ solution $\left(C_{\mathrm{NaCl}}=0.5 \mathrm{~m}, \mathrm{pH} 0\right.$, adjusted by adding $\mathrm{HCl}$ aqueous solution), respectively (Figure $3 \mathrm{f}$ and Supplementary Figure S10).

\section{Shape transformation in organic solvents}

Swelling of the PAA network made the Janus film 'smart' to achieve unidirectional bending with the PBMA network inwards. Likewise, the swelling of the PBMA network gives rise to bending of the Janus film with the PAA network inwards (Figure 4a). In organic solvents, such as cyclohexane, ethyl acetate, chloroform and toluene, the pure PBMA film exhibited a linear swelling ratio up to 1.7 (in toluene), whereas no obvious swelling of the pure PAA film was observed (Figure 4b). It is noteworthy that the PAA films shrunk in ethyl acetate due to its dissolving capacity for water and hardly changed in ethyl acetate saturated with water. Immersion in organic solvents (for example, toluene) caused the PAA-B-PBMA Janus film to curl into circles with the PAA network facing inwards due to the gradual 
a

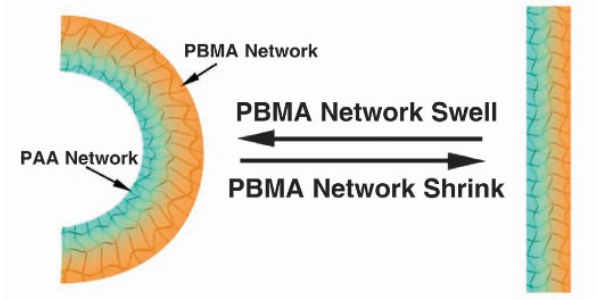

C

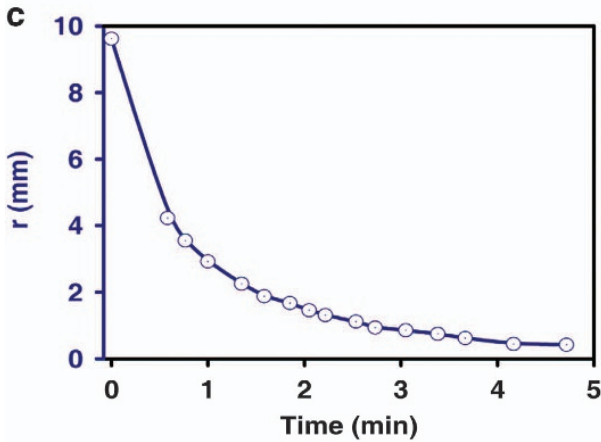

e

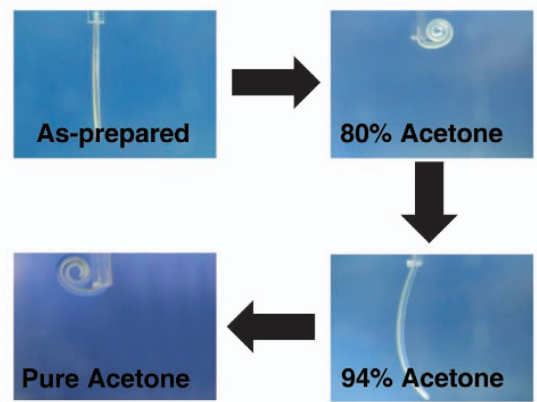

b

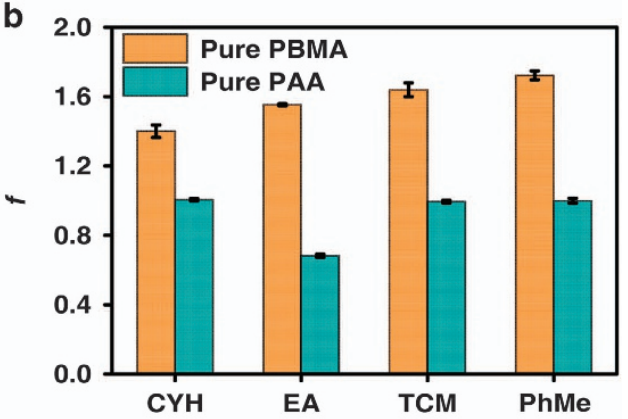

d

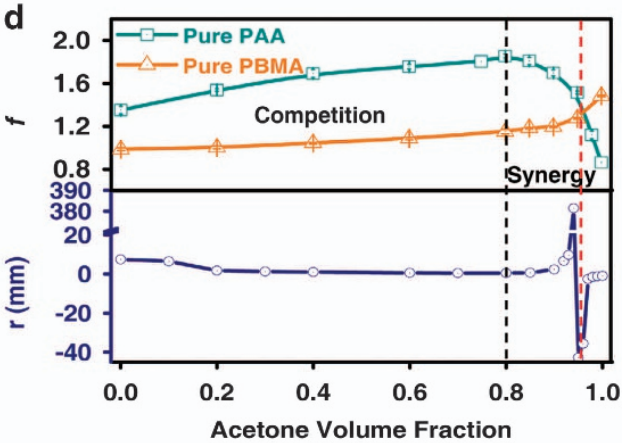

f

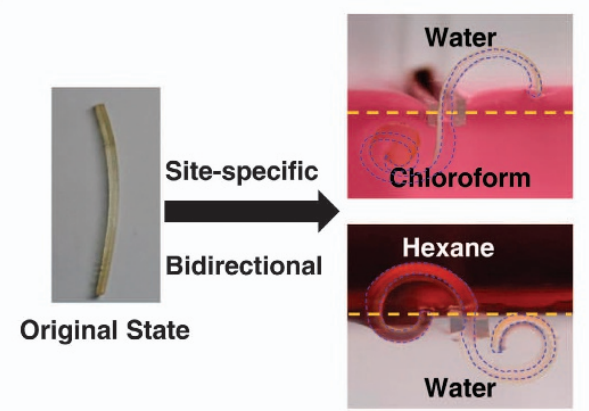

Figure 4 Shape transformation of the PAA-B-PBMA Janus film in the organic solvent and bidirectional, site-specific bending in composite solvent systems. (a) A schematic of the Janus film coiling and decoiling in organic solvents with the PBMA network as the active part. (b) Relative change in the linear dimensions, $f$, of the pure PAA and PBMA films in different organic solvents. (c) Bending of the PAA-B-PBMA Janus film in toluene and the plot of the radius of curvature against time. The radius of curvature reached a minimum within $5 \mathrm{~min}$. (d and e) Bidirectional bending of the Janus film in acetone-water mixtures under the binary cooperative effect of the hydrogel network and the organogel network. Plot of the linear dimensions, $f$, of the pure PAA and PBMA films ( $d$, top) and the radius of curvature of the PAA-B-PBMA film (d, bottom) against the acetone concentration. Typical images (e) of the PAA-B-PBMA Janus films in acetone-water mixtures with different concentrations. A straight PAA-B-PBMA film coiled gradually towards the PBMA network as the acetone volume fraction increases and reached its tightest at $\sim 80 \%$ concentration, due to the expansion competition between the PAA and the PBMA networks. The coiled film stretched again with a further increase of the acetone fraction from $80 \%$ to $94 \%$, during which the swelled PAA network shrunk and the PBMA network expanded. Further increasing the acetone fraction to $100 \%$, the film bent to the opposite direction with the PAA network facing inwards. (f) Site-specific bending of the Janus film. When immersed in aqueous solution/organic solvents systems, the Janus films displayed bidirectional bending with the water/oil interface as the cut-off point. In a hexane/water system, the upper part bent with the PAA inwards, whereas the bottom part bent with the PBMA inwards (f, top). In water/chloroform, the upper part bent with the PBMA inwards, whereas the bottom part bent with the PAA inwards (f, bottom). $\mathrm{CYH}$, cyclohexane; EA, ethyl acrylate; PhMe, toluene; TCM, chloroform.

increase of the internal stress in the Janus film with time arising from the swelling of the PBMA network (Figure $4 \mathrm{c}$ and Supplementary Figure S11 in ethyl acetate). In addition, reducing the thickness of the film can sharply increase the bending speed. By reducing the hydro/organo copolymer film to $170 \mu \mathrm{m}$, the time to bend into a circle $(r \approx 2 \mathrm{~mm}$ ) was shortened to $\sim 15 \mathrm{~s}$ from several minutes for films with a thickness of $1 \mathrm{~mm}$ (Supplementary Movie S2).

\section{Bidirectional bending}

Unidirectional bending, like pinecones, has been achieved in most artificial actuators, whereas bidirectional bending is still difficult to achieve, ${ }^{24,34,50}$ especially in a same solvent system. Here, bidirectional bending was achieved by the PAA-B-PBMA Janus film in acetone-water mixtures. When immersed in the acetone-water solution, the Janus films bent into circles with different radius of curvatures and directions depending on the acetone concentration (Supplementary Figure S12). In lower acetone concentrations $(<80 \%)$, the circles of the Janus films bent with the PBMA network facing inwards became smaller as the acetone concentration increased due to the increase in net difference in the linear swelling ratio $\left(\Delta f=f_{\mathrm{PAA}}-f_{\mathrm{PBMA}}\right)$ between the $\mathrm{PAA}$ and PBMA networks (Figure $4 \mathrm{~d}$, top). The minimum linear swelling ratio was reached at 


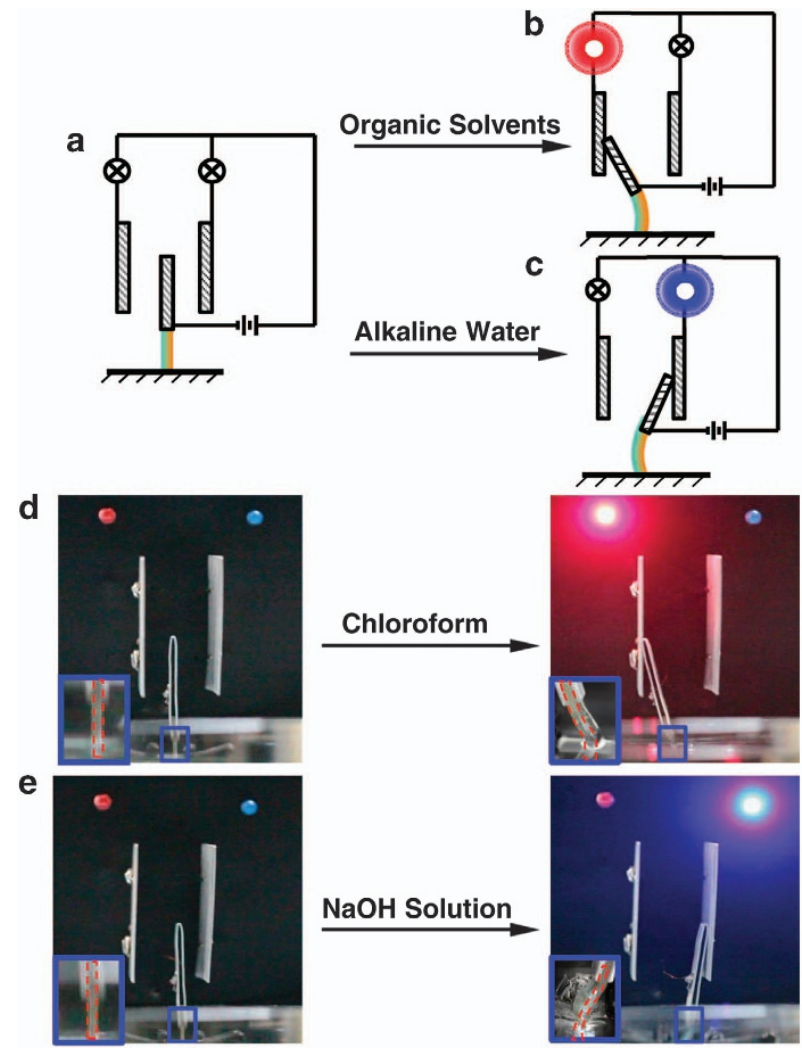

Figure 5 Smart sensor for solvents leakage detection. (a) Schematic representation of the smart sensor circuit. The PAA-B-PBMA Janus film with an electrode on the top works as a switch to sensitive to solvents. ( $b$ and $\mathbf{d}$ ) When organic solvents leak, with chloroform as an example, the films bent towards left with the PAA network facing inwards, as well as the electrode on the top of the film. Then, the left circuit was connected, lighting the red lamp on the left. (c and e) Similarly, the blue lamp on the right flashed when alkaline aqueous solution ( $\mathrm{NaOH}$ solution) was added, which is due to the film bending towards the right with the PBMA network facing inward, which closed the right circuit.

$\sim 80 \%$ acetone concentration (radius of curvature, $0.45 \mathrm{~mm}$ ), at which the PAA network swelled to its full extent (Figure $4 \mathrm{~d}$, bottom). Then, the binary cooperation between shrinking of the PAA network ${ }^{51,52}$ and the swelling of the PBMA network contributed to the decrease of $\Delta f$ with the increasing acetone concentration, even to negative values (Supplementary Figure S13). Accordingly, the curled PAA-B-PBMA Janus films stretched gradually (straight at 94\%, where $\Delta f \approx 0$ ) and bent to the opposite direction (represented as a negative value) with the PAA network facing inwards due to the negative $\Delta f$ (Figure $4 \mathrm{~d}$ and Supplementary Figure S14). As a result, under the binary cooperation between the hydrogel and organogel networks, the bending direction of the PAA-B-PBMA Janus film could be regulated in the acetone-water mixed solution system just by adjusting the acetone concentration (Figure 4e).

The PAA-B-PBMA Janus copolymer films exhibited excellent shape transformation properties in aqueous solutions and organic solvents. The changing bending direction of the Janus film has also been realized. In acetone aqueous solutions, the Janus film bent towards the PBMA side due to the swelling of the PAA networks. In ethyl acetate, the bent Janus film unbent gradually and bent towards the opposite direction (the PAA side) due to the swelling of the PBMA network and shrinking of the PAA network. In the acetone aqueous solution, the bent Janus film gradually stretched and bent towards the PBMA side again, whereby the PAA network swelled and the PBMA network shrunk. This process could be repeated multiple times with no effect on the bending performance of the Janus film (Supplementary Movie S3).

\section{Site-specific bending in an oil/water binary system}

The site-specific bending of the PAA-B-PBMA Janus film was also achieved in an oil/water binary system. Immersing the film in aqueous solution/organic solvent systems allows bidirectional bending simultaneously with a specific site as the cutoff point. In a hexane/water system, the PAA-B-PBMA Janus film bent towards the PAA network at the upper part of the system (in hexane) due to the swelling of the PBMA network, whereas bent towards the PBMA network at the bottom part of the system (in water) due to the PAA network swelling (Figure 4f, top). Similarly, in a water/chloroform system, the upper part of the Janus film bent with the PBMA network inwards (in water), whereas the bottom part film bent with the PAA network inwards (in chloroform) (Figure 4f, bottom) with the oil/water interface as cutoff point.

\section{Smart sensor for solvent leakage detection}

The unique sensitivity of the PAA-B-PBMA Janus film to most organic solvents and alkaline water endowed the film with the capability to work as a smart sensor for solvent leakage detection. As a proof-ofconcept, a sensor was designed and fabricated with the PAA-B-PBMA Janus film as sensing elements (Figure 5a). When an organic solvent (for example, chloroform) leaked, the Janus film bent towards the PAA network (left) resulting in the contact between the electrode at the top of the Janus film and the electrode plate on the left. After that, the left circuit was switched on, which brightened the warning lamp on the left (red light) (Figure 5b and d, and Supplementary Movie S4). By contrast, when alkaline water leaked, the Janus film bent towards the PBMA network (right) and the right circuit was closed, leading to the right warning lamp (blue light) being turned on (Figure $5 \mathrm{c}$ and e).

\section{CONCLUSION}

In conclusion, we successfully synthesized shape-transformable Janus films composed of a binary hydro/organo copolymer film via one-step polymerization of layered immiscible monomer solutions. Under the binary cooperative effect of the hydrogel network (low modulus) and the organogel network (high modulus), unidirectional bending of the PAA-B-PBMA Janus film was achieved by independently regulating the swelling of the PAA network or the PBMA network. Bidirectional bending was also realized in an acetone-water mixed solution system by utilizing the unique phase transition of the PAA network in acetone-water mixtures and site-specific bending was achieved in water/organic solvents systems. The directional bending to the external stimuli allowed the film to act as a smart sensor for solvent leakage detection. We believe that this interfacial copolymerization is applicable to most immiscible functional monomer systems (Supplementary Table S1 and Supplementary Figure S12), which will bring new perspectives and developments to functional polymer materials.

\section{CONFLICT OF INTEREST}

The authors declare no conflict of interest.

\section{ACKNOWLEDGEMENTS}

This research is supported by the National Natural Science Foundation (21425314, 21501184, 21434009 and 21421061), the Key Research Program of the Chinese Academy of Sciences (KJZD-EW-M01), the National High 
Technology Research and Development Program of China (863 Program) (2013AA032203), MOST (2013YQ190467) and the Top-Notch Young Talents Program of China.

1 Dawson, C., Vincent, J. F. V. \& Rocca, A.-M. How pine cones open. Nature 390, 668-668 (1997).

2 Forterre, Y., Skotheim, J. M., Dumais, J. \& Mahadevan, L. How the Venus flytrap snaps. Nature 433, 421-425 (2005).

3 Elbaum, R., Zaltzman, L., Burgert, I. \& Fratzl, P. The role of wheat awns in the seed dispersal unit. Science 316, 884-886 (2007).

4 Armon, S., Efrati, E., Kupferman, R. \& Sharon, E. Geometry and mechanics in the opening of chiral seed pods. Science 333, 1726-1730 (2011).

5 Burgert, I. \& Fratzl, P. Actuation systems in plants as prototypes for bioinspired devices. Phil. Trans. R. Soc. A 367, 1541-1557 (2009).

6 Harrington, M. J., Razghandi, K., Ditsch, F., Guiducci, L., Rueggeberg, M., Dunlop, J. W. C., Fratzl, P., Neinhuis, C. \& Burgert, I. Origami-like unfolding of hydro-actuated ice plant seed capsules. Nat. Commun. 2, 337 (2011).

7 Osada, Y., Okuzaki, H. \& Hori, H. A polymer gel with electrically driven motility. Nature 355, 242-244 (1992).

8 Pei, Q. \& Inganläs, O. Conjugated polymers and the bending cantilever method: electrical muscles and smart devices. Adv. Mater. 4, 277-278 (1992).

9 Otero, T. F. \& Sansieña, J. M. Soft and wet conducting polymers for artificial muscles. Adv. Mater. 10, 491-494 (1998)

10 Takashima, Y., Hatanaka, S., Otsubo, M., Nakahata, M., Kakuta, T., Hashidzume, A., Yamaguchi, H. \& Harada, A. Expansion-contraction of photoresponsive artificial muscle regulated by host-guest interactions. Nat. Commun. 3, 1270 (2012).

11 Chun, K.-Y., Kim, S. H., Shin, M. K., Kwon, C. H., Park, J., Kim, Y. T., Spinks, G. M., Lima, M. D., Haines, C. S., Baughman, R. H. \& Kim, S. J. Hybrid carbon nanotube yarn artificial muscle inspired by spider dragline silk. Nat. Commun. 5, 3322 (2014).

12 Haines, C. S., Lima, M. D., Li, N., Spinks, G. M., Foroughi, J., Madden, J. D. W., Kim, S. H., Fang, S., Jung de Andrade, M., Göktepe, F., Göktepe, Ö., Mirvakili, S. M., Naficy, S., Lepró, X., Oh, J., Kozlov, M. E., Kim, S. J., Xu, X., Swedlove, B. J., Wallace, G. G. \& Baughman, R. H. Artificial muscles from fishing line and sewing thread. Science 343, 868-872 (2014).

13 lamsaard, S., Aßhoff, S. J., Matt, B., Kudernac, T., CornelissenJeroen, J. L. M., Fletcher, S. P. \& Katsonis, N. Conversion of light into macroscopic helical motion. Nat. Chem. 6, 229-235 (2014).

14 Smela, E., Inganäs, O. \& Lundström, I. Controlled folding of micrometer-size structures. Science 268, 1735-1738 (1995).

15 Jager, E. W. H., Inganäs, O. \& Lundström, I. Microrobots for micrometer-size objects in aqueous media: potential tools for single-cell manipulation. Science 288, 2335-2338 (2000)

16 Maeda, S., Hara, Y., Sakai, T., Yoshida, R. \& Hashimoto, S. Self-walking gel. Adv. Mater. 19, 3480-3484 (2007).

17 Ilievski, F., Mazzeo, A. D., Shepherd, R. F., Chen, X. \& Whitesides, G. M. Soft robotics for chemists. Angew. Chem. Int. Ed. 50, 1890-1895 (2011).

18 Palleau, E., Morales, D., Dickey, M. D. \& Velev, O. D. Reversible patterning and actuation of hydrogels by electrically assisted ionoprinting. Nat. Commun. 4, 2257 (2013).

19 Magdanz, V., Stoychev, G., Ionov, L., Sanchez, S. \& Schmidt, O. Stimuli-responsive microjets with reconfigurable shape. Angew. Chem. Int. Ed. 126, 2711-2715 (2014).

20 Zhang, L., Liang, H., Jacob, J. \& Naumov, P. Photogated humidity-driven motility. Nat. Commun. 6, 7429 (2015).

21 Klein, Y., Efrati, E. \& Sharon, E. Shaping of elastic sheets by prescription of non-euclidean metrics. Science 315, 1116-1120 (2007).

22 Kim, J., Hanna, J. A., Byun, M., Santangelo, C. D. \& Hayward, R. C. Designing responsive buckled surfaces by halftone gel lithography. Science 335, 1201-1205 (2012).

$23 \mathrm{Hu}, \mathrm{Z}$., Zhang, X. \& Li, Y. Synthesis and application of modulated polymer gels. Science 269, 525-527 (1995).

24 Erb, R. M., Sander, J. S., Grisch, R. \& Studart, A. R. Self-shaping composites with programmable bioinspired microstructures. Nat. Commun. 4, 1712 (2013).

25 Zhang, X., Yu, Z., Wang, C., Zarrouk, D., Seo, J.-W. T., Cheng, J. C., Buchan, A. D., Takei, K., Zhao, Y., Ager, J. W., Zhang, J., Hettick, M., Hersam, M. C., Pisano, A. P., Fearing, R. S. \& Javey, A. Photoactuators and motors based on carbon nanotubes with selective chirality distributions. Nat. Commun. 5, 2983 (2014).

26 Wu, Z. L., Moshe, M., Greener, J., Therien-Aubin, H., Nie, Z., Sharon, E. \& Kumacheva, E. Three-dimensional shape transformations of hydrogel sheets induced by small-scale modulation of internal stresses. Nat. Commun. 4, 1586 (2013).
27 Yu, Y., Nakano, M. \& Ikeda, T. Photomechanics: directed bending of a polymer film by light. Nature 425, 145-145 (2003).

28 Morin, S. A., Shepherd, R. F., Kwok, S. W., Stokes, A. A., Nemiroski, A. \& Whitesides, G. M. Camouflage and display for soft machines. Science 337, 828-832 (2012).

29 Stoychev, G., Zakharchenko, S., Turcaud, S., Dunlop, J. W. C. \& Ionov, L. Shape-programmed folding of stimuli-responsive polymer bilayers. ACS Nano 6 , 3925-3934 (2012).

30 Stoychev, G., Turcaud, S., Dunlop, J. W. C. \& Ionov, L. Hierarchical multi-step folding of polymer bilayers. Adv. Funct. Mater. 23, 2295-2300 (2013).

31 Gladman, A. S., Matsumoto, E. A., Nuzzo, R. G., Mahadevan, L. \& Lewis, J. A. Biomimetic 4D printing. Nat. Mater. 15, 413-418 (2016).

32 Stoychev, G., Puretskiy, N. \& Ionov, L. Self-folding all-polymer thermoresponsive microcapsules. Soft Matter 7, 3277-3279 (2011).

$33 \mathrm{Ma}$, M., Guo, L., Anderson, D. G. \& Langer, R. Bio-inspired polymer composite actuator and generator driven by water gradients. Science 339, 186-189 (2013).

34 Baughman, R. H., Cui, C., Zakhidov, A. A., Iqbal, Z., Barisci, J. N., Spinks, G. M., Wallace, G. G., Mazzoldi, A., De Rossi, D., Rinzler, A. G., Jaschinski, O., Roth, S. \& Kertesz, M. Carbon nanotube actuators. Science 284, 1340-1344 (1999).

35 Ionov, L. Polymeric actuators. Langmuir 31, 5015-5024 (2015).

36 Forster, S. \& Antonietti, M. Amphiphilic block copolymers in structure-controlled nanomaterial hybrids. Adv. Mater. 10, 195-217 (1998).

37 Jiang, L., Wang, R., Yang, B., Li, T. J., Tryk, D. A., Fujishima, A., Hashimoto, K. \& Zhu, D. B. Binary cooperative complementary nanoscale interfacial materials. Pure Appl. Chem. 72, 73-81 (2000).

$38 \mathrm{Su}, \mathrm{B} ., \mathrm{Guo}$, W. \& Jiang, L. Learning from nature: binary cooperative complementary nanomaterials. Small 11, 1072-1096 (2015).

39 Discher, D. E. \& Eisenberg, A. Polymer vesicles. Science 297, 967-973 (2002).

40 Cornelissen, J. J. L. M., Fischer, M., Sommerdijk, N. A. J. M. \& Nolte, R. J. M. Helical superstructures from charged poly(styrene)-poly(isocyanodipeptide) block copolymers. Science 280, 1427-1430 (1998).

41 Letchford, K. \& Burt, H. A review of the formation and classification of amphiphilic block copolymer nanoparticulate structures: micelles, nanospheres, nanocapsules and polymersomes. Eur. J. Pharm. Biopharm. 65, 259-269 (2007).

42 Blanazs, A., Madsen, J., Battaglia, G., Ryan, A. J. \& Armes, S. P. Mechanistic insights for block copolymer morphologies: how do worms form vesicles? J. Am. Chem. Soc. 133, 16581-16587 (2011).

43 Velonia, K., Rowan, A. E. \& Nolte, R. J. M. Lipase polystyrene giant amphiphiles. J. Am. Chem. Soc. 124, 4224-4225 (2002).

44 Discher, B. M., Won, Y. Y., Ege, D. S., Lee, J. C. M., Bates, F. S., Discher, D. E. \& Hammer, D. A. Polymersomes: tough vesicles made from diblock copolymers. Science 284, 1143-1146 (1999).

45 Shimizu, T., Masuda, M. \& Minamikawa, H. Supramolecular nanotube architectures based on amphiphilic molecules. Chem. Rev. 105, 1401-1444 (2005).

$46 \mathrm{La}$, Y., Park, C., Shin, T. J., Joo, S. H., Kang, S. \& Kim, K. T. Colloidal inverse bicontinuous cubic membranes of block copolymers with tunable surface functional groups. Nat. Chem. 6, 534-541 (2014).

47 Timoshenko, S. Analysis of bi-metal thermostats. J. Opt. Soc. Am. 11, 233-255 (1925)

48 Ohmine, I. \& Tanaka, T. Salt effects on the phase transition of ionic gels. J. Chem. Phys. 77, 5725-5729 (1982).

49 Huther, A., Schafer, B., Xu, X. \& Maurer, G. Phase equilibria of hydrogel systems. Phys. Chem. Chem. Phys. 4, 835-844 (2002).

$50 \mathrm{Ma}, \mathrm{C} ., \mathrm{Li}, \mathrm{T} .$, Zhao, Q., Yang, X., Wu, J., Luo, Y. \& Xie, T. Supramolecular lego assembly towards three-dimensional multi-responsive hydrogels. Adv. Mater. 26, 5665-5669 (2014)

51 Tanaka, T. Collapse of gels and critical endpoint. Phys. Rev. Lett. 40, 820-823 (1978).

52 IImain, F., Tanaka, T. \& Kokufuta, E. Volume transition in a gel driven by hydrogenbonding. Nature 349, 400-401 (1991).

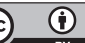

This work is licensed under a Creative Commons Attribution 4.0 International License. The images or other third party material in this article are included in the article's Creative Commons license, unless indicated otherwise in the credit line; if the material is not included under the Creative Commons license, users will need to obtain permission from the license holder to reproduce the material. To view a copy of this license, visit http:// creativecommons.org/licenses/by/4.0/

(C) The Author(s) 2017

Supplementary Information accompanies the paper on the NPG Asia Materials website (http://www.nature.com/am) 